\title{
STRESS-STRAIN DISTRIBUTION IN THE HUMAN STOMACH
}

\author{
N.R. AKHMADEEV ${ }^{1} \&$ R. MIFTAHOF 2 \\ ${ }^{1}$ Kazan Medical University, Kazan, Tatarstan, Russian Federation. \\ ${ }^{2}$ Arabian Gulf University, Kingdom of Bahrain.
}

\begin{abstract}
A biomechanical model of the human stomach is proposed, that is based on detailed biological data of the structure and function of the organ. The process of electromechanical conjugation and the spread of the electromechanical wave along the stomach wall were analyzed numerically. Results revealed patterns of stress-strain distribution in the organ. Thus the fundus, the body and the antrum of the organ always experience biaxial stress-strain states, while the cardia and the pylorus undergo uniaxial loading. The circular smooth muscle layer produced greater total forces throughout in comparison to the outer longitudinal smooth muscle layer. The body of the organ along the lesser curvature and the cardia-fundus areas were overstressed compare to other regions. Although the theoretical results resemble qualitatively patterns of electrical and mechanical activity observed in vivo and in vitro there is currently no affirmative experimental evidence to provide a detailed quantitative comparison of the results.

Keywords: mathematical model, human stomach, numerical simulations, electromechanical activity, peristalsis
\end{abstract}

\section{INTRODUCTION}

High endurance and enormous functionality of abdominal viscera depend on biomechanical properties of the tissues they are made of and specific arrangements of internal constituents. Soft biological tissues, without exception, are regarded as anisotropic heterogeneous incompressible composites that possess nonlinear viscoelastic mechanical characteristics [1,2]. The distinctive anatomical appearance of organs is strongly influenced by the force systems to which they are subjected and is correlated with their structural advantages. The stomach, the small and large intestines share these properties. For example, the stomach is the organ of the gastrointestinal tract that is located in the left upper quadrant of the abdomen immediately below the diaphragm. Its prime role is to accommodate and digest ingested food. Even at small thickness of the wall, which in normal subjects varies from 3 to $5 \mathrm{~mm}$, and the characteristic radius of the curvature of the middle surface ranging within $10 \leq R_{i} \leq 15(\mathrm{~cm})$ it is capable of holding 2-5 1 of mixed gastric content without significantly increasing intraluminal pressure [3].

Mathematical modeling allows one to gain the insight into hidden physiological mechanisms of function of experimentally inaccessible abdominal viscera [4-10]. However, most of the proposed models are based on a reductionist 'mechanistic' approach and, therefore, have a limited biological plausibility. Furthermore, these studies have been hampered by indiscrete and often inappropriate applications of ideas and methods borrowed from the mechanics of shells to describe their biomechanical behavior. It should be emphasized that biomechanics is not just the transformation of general laws and principles of mechanics to the study of biological phenomena, but rather the adequate development and extension of these laws and principles to the modeling and analysis of living things. Integrative models that incorporate multilevel data and serve as the basis for complex analysis of interrelated biological processes at the ion channel, cellular, tissue and organ levels are needed [11, 12]. Such models will have enormous implications for our understanding of the pathophysiology of various diseases and their pharmacological treatment.

The aims of this study are: (i) to formulate a biomechanical model of the human stomach as a thin soft biological shell, and (ii) to study the dynamics of the propagation of electromechanical waves within the organ under normal physiological conditions.

(C) 2010 WIT Press, www.witpress.com

ISSN: 1755-7437 (paper format), ISSN: 1755-7445 (online), http://journals.witpress.com

DOI: 10.2495/DNE-V5-N2-90-107 


\section{PHYSIOLOGY OF THE STOMACH}

The configuration of the stomach is determined by: (i) the amount of the content, (ii) the stage of the digestive process, (iii) the degree of development of the gastric musculature, and (iv) the condition of the adjacent loops of the small and large intestines. Anatomically, the stomach is concave on its right side, convex on its left. The concave border is called the lesser curvature; the convex border, the greater curvature. The region that connects the lower esophagus with the upper part of the stomach is called the cardia. The uppermost adjacent part to it is the fundus. The fundus adapts to the varying volume of ingested food and it frequently contains a gas bubble, especially after a meal. The largest part of the stomach is known simply as the body. It functions as a reservoir for ingested food and liquids. The antrum, the lowermost part of the stomach, is usually funnel-shaped, with its narrow end connecting with the pyloric region. The latter empties into the duodenum - the upper division of the small intestine. The pyloric portion of the stomach tends to curve to the right and slightly upward and backward and thus gives the stomach its J-shaped appearance.

Physiological responses of the stomach to internal and external stimuli depend entirely on the inherent activity of histomorphological elements: smooth muscle cells, neurons, interstitial cells of Cajal, and their topographical organization in gastric tissue [13-16]. Longitudinal and circular smooth muscle layers, formed of smooth muscle cells, are embedded into a network of collagenous and elastin fibers and are coupled via gap junctions into three distinct syncytia (muscle layers). The external longitudinal muscle layer continues from the esophagus into the duodenum. The middle uniform circular layer is the strongest and completely covers the stomach. The circular fibers are best developed in the antrum and pylorus. At the pyloric end, the circular muscle layer greatly thickens to form the pyloric sphincter. The innermost oblique muscular layer is limited chiefly to the cardia-fundal regions and progressively weakens as it approaches the pylorus.

The smooth muscle syncytia react with a variety of rhythmical movements which are a result of an electrochemical coupling phenomenon. Electrophysiological and molecular cloning studies have shown that L- and T-type $\mathrm{Ca}^{2+}$ channels, $\mathrm{Ca}^{2+}$-activated $\mathrm{K}^{+}$, selective $\mathrm{K}^{+}, \mathrm{Na}^{+}$, leak $\mathrm{Cl}^{-}$, and nonselective cation channels are responsible for electrical activity of smooth muscle syncytia represented by slow waves and spikes [17-20]. Slow waves are low amplitude, 20-38 mV membrane potential fluctuations, and spikes are high amplitude, $35-45 \mathrm{mV}$, with action potentials occurring on the crests of slow waves. The role of pacemakers belongs to interstitial cells of Cajal, which are located within the myenteric nervous plexus and smooth muscle syncytia [21-23]. They are coupled to the syncytium via gap junctions. Contractions in the smooth muscle are initiated by myosin light chain phosphorylation via the activation of $\mathrm{Ca}^{2+}$ calmodulin-dependent myosin light chain-kinase. The key player in the process is free cytoplasmic $\mathrm{Ca}^{2+}$. In the resting state, the concentration of intracellular $\mathrm{Ca}^{2+}$ is $\approx 0.1 \mu \mathrm{M}$ which upon stimulation rises to high levels as a result of influx through $\mathrm{L}$ - and T-type $\mathrm{Ca}^{2+}$ channels, and induced calcium release from the sarcoplasmic reticulum [24].

Connective tissue elastin and collagen fibers are built in a three dimensional loosely woven network. Elastin may be stretched to $250 \%$ of the unloaded configuration. Collagen is relatively inextensible stiff reinforcing structural component, and the main load carrying element. Collagen fibers are usually undulated and they become stiff when straightened under the action of applied loads. The strength of tissues is strongly correlated with the collagen content [1]. The uttermost tunica serosa coats the entire organ and provides its final shape.

The mucous membrane of the stomach consists of epithelial and glandular cells. Their main function is digestion, solute transport, protection and immune response and their role in load distribution is negligible.

Motility signals in the stomach originate in the upper part of the body of the organ. Three types of mechanical waves are observed: (i) small isolated contraction waves, (ii) peristaltic waves that 
slowly move from the point of origin down toward the pyloric sphincter, and (iii) nonpropagating waves. Contractions produce slight or deep indentations in the wall and serve as mixing, crushing and pumping mechanisms for the gastric contents [25].

\section{MECHANICAL PROPERTIES OF THE TISSUE}

The soft tissue that makes the wall of the stomach is characterized as transversely anisotropic nonhomogenous viscoelastic biocomposite that undergoes finite strains. This has been convincingly demonstrated in quasistatic uniaxial tests on specimens collected from different regions of the organ $[26,27]$. The force-ratio of elongation $(T-\lambda)$ data indicate that the tissue is initially compliant, $1.0<$ $\lambda \leq 1.3-1.4$, and stiffens at higher loads. For $1.4<\lambda \leq 2.0$ the tissue behaves nearly elastically until it breaks at $T_{\max }=1.5 \mathrm{~N} / \mathrm{cm}$. Stretching of specimens excised in the directions of the longitudinal, circumferential and oblique layers has shown properties of transverse anisotropy. The wall has lower extensibility along the orientation of longitudinal smooth muscle fibers and the highest along the circumferential layer. Experiments under biaxial tension have shown that the shear force applied to the tissue is significantly less, $0.01 T_{\max }$, compared with the tangential force [28].

Microscopic analysis of the dynamics of crack nucleation and growth within the wall of the stomach has revealed that first small randomly oriented cracks appear in the submucosal and mucous layers at $1.0<\lambda \leq 1.1$ [29]. They align perpendicular to the axis of the stretch force as $\lambda$ increases. The fracture of entire layers occurs at $\lambda=1.3-1.4$ and $T=0.1 \mathrm{~N} / \mathrm{cm}$. The most extensible and resistible to loads are the connective tissue network of elastin and collagen fibers, and smooth muscle syncytia. They elongate up to a maximum $\lambda_{\max }=2.2-2.5$ and withstand the maximum force of $T=1.5-2.0 \mathrm{~N} / \mathrm{cm}$ before the complete break.

The complex behavior of the stomach has also been studied under complex loading, i.e. a combined inflation with subsequent external local compression of the anterior surface of the organ [30]. Instantaneous intraluminal pressure, volume and stress-strain recordings indicate that in the low pressure domain $0.1 \leq P \leq 3.0(\mathrm{kPa})$ the stomach experiences biaxial stress-strain states and smooth continuous deformations in all regions. In the higher pressure range $3.0<P \leq 25(\mathrm{kPa})$ creases appear in the cardia-fundal and antrum-pyloric areas.

\section{MODEL ASSUMPTIONS}

A biomechanical model of the stomach is based on the following anatomical, physiological and mechanical assumptions of its appearance and function:

1. The stomach is a closed thin soft shell of complex geometry; it satisfies the criterion of thin shells: $\max \left(h / R_{i}\right) \leq 1 / 20$, where $h$ is the thickness and $R_{i}$ are the radii of the curvature of the middle surface of the shell, and the conditions of softness: the wall of stomach does not resist compression and bending, the shear stresses are negligibly small, and creases may be formed during deformation.

2. The wall of the bioshell (stomach) is formed of two distinct longitudinal and circular smooth muscle layers embedded into a network of elastin and collagen fibers; the layers display an orthogonal type of weaving in the undeformed configuration.

3. The biocomposite is a nonlinear viscoelastic transversely anisotropic continuum undergoing finite strains; its 'passive' component depends on the mechanics of inactive smooth muscle syncytia, collagen and elastin fibers; the 'active' component - the generation of forces of contraction and relaxation, is the result of electromechanical conjugation at the level of intracellular contractile proteins; the key biochemical factor in the process of conjugation is free $\mathrm{Ca}^{2+}$ ions.

4. Muscle layers are electrically excitable biological media; the membrane potentials are due to the dynamics of $\mathrm{Ca}^{2+}, \mathrm{Na}^{+}, \mathrm{K}^{+}$and $\mathrm{Cl}^{-}$channels; the longitudinal smooth muscle layer possesses anisotropic cable electrical characteristics, while the circular layer is electrically isotropic. 
5. The initial conditions assume that the stomach is in the resting unexcited state; a single pacemaker or multiple spatially distributed pacemakers are associated with interstitial cells of Cajal; their discharge rates and intensity are assumed to be known a priori; they provide excitatory signals to the system.

6. Clamped edge boundary conditions are realized at the cardiac and pyloric ends of the shell throughout simulations.

Let every point of the middle surface of the undeformed stomach be referred to the global Cartesian coordinate system $x, y, z$. Assume that the organ is inflated by intraluminal pressure $p$ and subsequently excited by electrical discharges of the pacemaker cell of a given intensity and duration. As a result of depolarization the electrical waves are produced in the longitudinal and circular smooth muscle syncytia. They propagate along the surface of the organ and generate active forces of contraction-relaxation.

Mathematical formulation of the problem of the dynamics of electromechanical wave activity in the stomach comprises the equations of motion of the thin soft shell, constitutive relations for mechanical and cable electrical properties of the biocomposite, initial and boundary conditions. Thus, the equations of motion of the soft biological shell are given by

$$
\begin{aligned}
\rho_{0} \frac{\partial v_{x}}{\partial t}= & \frac{\partial}{\partial \tilde{s}_{1}}\left[\left(k \frac{\partial\left(\lambda_{c}-1\right)}{\partial t}+T^{a}\left(\lambda_{c}\right)+T^{p}\left(\lambda_{c}, \lambda_{l}\right)\right) e_{1 x} \sqrt{a_{22}}\right] \\
& +\frac{\partial}{\partial \tilde{s}_{2}}\left[\left(k \frac{\partial\left(\lambda_{l}-1\right)}{\partial t}+T^{a}\left(\lambda_{l}\right)+T^{p}\left(\lambda_{c}, \lambda_{l}\right)\right) e_{2 x} \sqrt{a_{11}}\right]+p \sqrt{a} \bar{n}_{x}, \\
\rho_{0} \frac{\partial v_{y}}{\partial t}= & \frac{\partial}{\partial \tilde{s}_{1}}\left[\left(k \frac{\partial\left(\lambda_{c}-1\right)}{\partial t}+T^{a}\left(\lambda_{c}\right)+T^{p}\left(\lambda_{c}, \lambda_{l}\right)\right) e_{1 y} \sqrt{a_{22}}\right] \\
& +\frac{\partial}{\partial \tilde{s}_{2}}\left[\left(k \frac{\partial\left(\lambda_{l}-1\right)}{\partial t}+T^{a}\left(\lambda_{l}\right)+T^{p}\left(\lambda_{c}, \lambda_{l}\right)\right) e_{2 y} \sqrt{a_{11}}\right]+p \sqrt{a} \bar{n}_{y}, \\
\rho_{0} \frac{\partial v_{z}}{\partial t}= & \frac{\partial}{\partial \tilde{s}_{1}}\left[\left(k \frac{\partial\left(\lambda_{c}-1\right)}{\partial t}+T^{a}\left(\lambda_{c}\right)+T^{p}\left(\lambda_{c}, \lambda_{l}\right)\right) e_{1 z} \sqrt{a_{22}}\right] \\
& +\frac{\partial}{\partial \tilde{s}_{2}}\left[\left(k \frac{\partial\left(\lambda_{l}-1\right)}{\partial t}+T^{a}\left(\lambda_{l}\right)+T^{p}\left(\lambda_{c}, \lambda_{l}\right)\right) e_{2 z} \sqrt{a_{11}}\right]+p \sqrt{a} \overline{n_{z}},
\end{aligned}
$$

where $\tilde{s}_{1}, \tilde{s}_{2}$ are the Lagrangian curvilinear orthogonal coordinates and $t$ is time. The components of the velocity vector $v_{x}, v_{y}$ and $v_{z}$ are

$$
v_{x}=\frac{d x}{d t}, \quad v_{y}=\frac{d y}{d t}, \quad v_{z}=\frac{d z}{d t}
$$

The rate of elongation (hereafter, the subscripts $(l)$ and $(c)$ are referred to the longitudinal and circular muscle layers, respectively), are calculated as

$$
\lambda_{c}=\frac{d x}{d \dot{\circ}}, \quad \lambda_{l}=\frac{d y}{d \dot{\circ}} .
$$

The components and the determinant of the metric tensor are defined by

$$
\begin{aligned}
a_{i j} & =\frac{\partial x}{\partial \tilde{s}_{i}} \frac{\partial x}{\partial \tilde{s}_{j}}+\frac{\partial y}{\partial \tilde{s}_{i}} \frac{\partial y}{\partial \tilde{s}_{j}}+\frac{\partial z}{\partial \tilde{s}_{i}} \frac{\partial z}{\partial \tilde{s}_{j}} \\
a & =a_{11} a_{22}-a_{12}^{2} \quad(i=1,2)
\end{aligned}
$$

and the cosines of the outward normal $\bar{n}$ to the surface with respect to the $x, y, z$ axes are calculated as 


$$
\begin{gathered}
e_{i x}=\frac{1}{\sqrt{a_{i i}}} \frac{\partial x}{\partial \tilde{s}_{i}}, \quad e_{i y}=\frac{1}{\sqrt{a_{i i}}} \frac{\partial y}{\partial \tilde{s}_{i}}, \quad e_{i z}=\frac{1}{\sqrt{a_{i i}}} \frac{\partial z}{\partial \tilde{s}_{i}}, \\
\bar{n}_{x}=\left(e_{1 y} e_{2 y}-e_{1 z} e_{2 z}\right) \sqrt{a_{11} a_{22}} / \sqrt{a}, \\
\bar{n}_{y}=\left(e_{1 z} e_{2 x}-e_{1 x} e_{2 s}\right) \sqrt{a_{11} a_{22}} / \sqrt{a}, \\
\bar{n}_{z}=\left(e_{1 x} e_{2 y}-e_{1 y} e_{2 x}\right) \sqrt{a_{11} a_{22}} / \sqrt{a} .
\end{gathered}
$$

The passive $T_{c, l}^{p}$ and active $T_{c, l}^{a}$ forces of the total membrane force are given by

$$
\begin{gathered}
T_{c, l}^{p}=\frac{\partial \rho_{0} W}{\partial\left(\lambda_{c, l}-1\right)}, \\
T_{c, l}^{a}=\left\{\begin{array}{l}
0, \quad\left[\mathrm{Ca}^{2+}\right] \leq 0.1 \mu \mathrm{M} \\
c_{7}+c_{8}\left[\mathrm{Ca}^{2+}\right]^{4}+c_{9}\left[\mathrm{Ca}^{2+}\right]^{3}+c_{10}\left[\mathrm{Ca}^{2+}\right]^{2}+c_{11}\left[\mathrm{Ca}^{2+}\right], \quad 0.1<\left[\mathrm{Ca}^{2+}\right] \leq 1 \mu \mathrm{M} \\
\max T^{a}, \quad\left[\mathrm{Ca}^{2+}\right]>1 \mu \mathrm{M}
\end{array}\right.
\end{gathered}
$$

where $W$ is the strain energy density function of the connective tissue network and passive muscle tissue

$$
\begin{aligned}
\rho_{0} W= & \frac{1}{2}\left[c_{1}\left(\lambda_{l}-1\right)^{2}+2 c_{3}\left(\lambda_{l}-1\right)\left(\lambda_{c}-1\right)+c_{2}\left(\lambda_{c}-1\right)^{2}\right. \\
& \left.+c_{14} \exp \left(c_{4}\left(\lambda_{l}-1\right)^{2}+c_{5}\left(\lambda_{c}-1\right)^{2}+2 c_{6}\left(\lambda_{l}-1\right)\left(\lambda_{c}-1\right)\right)\right]
\end{aligned}
$$

Here $c_{1-11}$ are empirically defined mechanical constants, and $\left[\mathrm{Ca}^{2+}\right]$ is the concentration of free intracellular calcium.

The characteristic feature of thin soft shells is the possibility of the simultaneous coexistence of smooth (biaxially stress) and wrinkled (uniaxially stress) zones. The smooth surface is characterized by $\lambda_{l, c}>1.0\left(T_{l, c}>0\right)$; in a case of the development of wrinkles, either $\lambda_{1} \leq 1.0, \lambda_{c} \leq 1.0\left(T_{l}=0, T_{c}>\right.$ $0)$, or $\lambda_{\mathrm{c}} \leq 1.0, \lambda_{l}>1.0\left(T_{l}>0, T_{c}=0\right)$. The wrinkled zone is substituted by an ironed out zone made of a set of unbound muscle and connective tissue fibers aligned with the direction of positive tensile stress. This is determined by general requirements of the conservation of smoothness of the surface and the continuity of membrane forces of the shell. Thus, if creases appear then the shell is subjected to the uniaxial stress-strained state. Therefore, for $T_{c, l}^{p}$ we have

$$
T_{(c, l)}^{p}=\left\{\begin{array}{l}
0, \quad \lambda_{(c, l)} \leq 1, \\
c_{12}\left[\exp \left(c_{13}\left(\lambda_{(c, l)}-1\right)-1\right], \quad \lambda_{(c, l)}>1 .\right.
\end{array}\right.
$$

Changes in the free cytosolic calcium concentration yield

$$
d\left[\mathrm{Ca}^{2+}\right] / d t=0.2 z\left(\varphi_{\mathrm{Ca}}-\varphi_{(c, l)}\right) /\left(1+\zeta\left[\mathrm{Ca}^{2+}\right]\right)-0.3\left[\mathrm{Ca}^{2+}\right]
$$

Here $\varphi_{\mathrm{Ca}}$ is the reversal potential for the calcium ion current; $\zeta$ is the parameter of inhibition of the $\mathrm{Ca}^{2+}$ channels; $z$ is the dynamic variable of the ionic channels whose kinetics is given by

$$
\frac{d z}{d t}=\left(\frac{1}{1+\exp \left(-0.15\left(\varphi_{(c, l)}+42\right)\right.}-z\right) / \tau_{z},
$$

$\tau_{z}$ is the time constant. 
The dynamics of the propagation of the electrical wave, $\varphi_{l}$, along the anisotropic longitudinal muscle layer is given by

$$
C_{m} \frac{\partial \varphi_{l}}{\partial t}=I_{m 1}\left(\tilde{s}_{1}, \tilde{s}_{2}\right)+I_{m 2}\left(\tilde{s}_{1}-\tilde{s}_{1}^{\prime}, \tilde{s}_{2}-\tilde{s}_{2}^{\prime}\right)+I_{\text {ionic }}^{*},
$$

where $I_{m 1}, I_{m 2}$ are the transmembrane currents

$$
\begin{aligned}
& I_{m 1}\left(\tilde{s}_{1}, \tilde{s}_{2}\right)=M_{v s}\left\{-\frac{2\left(\mu_{\tilde{s}_{1}}-\mu_{\tilde{s}_{2}}\right)}{\left(1+\mu_{\tilde{s}_{1}}\right)\left(1+\mu_{\tilde{s}_{1}}\right)} \arctan \left(\frac{d \tilde{s}_{1}}{d \tilde{s}_{2}} \sqrt{\frac{G_{\tilde{s}_{2}}}{G_{\tilde{s}_{1}}}}\right)+\frac{g_{0, \tilde{s}_{2}}^{*}}{G_{\tilde{s}_{1}}}\right\} \\
& \times\left(\frac{\partial}{\partial \tilde{s}_{1}}\left(\frac{g_{0, \tilde{s}_{1}}^{*}}{\lambda_{c}} \frac{\partial \varphi_{1}}{\partial \tilde{s}_{1}}\right)+\frac{\partial}{\partial \tilde{s}_{2}}\left(\frac{g_{0, \tilde{s}_{2}}^{*}}{\lambda_{l}} \frac{\partial \varphi_{1}}{\partial \tilde{s}_{2}}\right)\right), \\
& I_{m 2}\left(\tilde{s}_{1}, \tilde{s}_{2}\right)=M_{v s} \iint_{S} \frac{\mu_{\tilde{s}_{1}}-\mu_{\tilde{s}_{2}}}{2 \pi\left(1+\mu_{\tilde{s}_{1}}\right)\left(1+\mu_{\tilde{s}_{1}}\right)} \frac{\left(\tilde{s}_{2}-\tilde{s}_{2}^{\prime}\right) / G_{\tilde{s}_{2}}-\left(\tilde{s}_{1}-\tilde{s}_{1}^{\prime}\right) / G_{\tilde{s}_{1}}}{\left[\left(\tilde{s}_{1}-\tilde{s}_{1}^{\prime}\right) / G_{\tilde{s}_{1}}-\left(\tilde{s}_{2}-\tilde{s}_{2}^{\prime}\right) / G_{\tilde{s}_{2}}\right]^{2}} \\
& \times\left(\frac{\partial}{\partial \tilde{s}_{1}}\left(\frac{g_{0, \tilde{s}_{1}}^{*}}{\lambda_{c}} \frac{\partial \varphi_{l}}{\partial \tilde{s}_{1}}\right)+\frac{\partial}{\partial \tilde{s}_{2}}\left(\frac{g_{0, \tilde{s}_{2}}^{*}}{\lambda_{l}} \frac{\partial \varphi_{l}}{\partial \tilde{s}_{2}}\right)\right) d \tilde{s}_{1}^{\prime}{ }_{1} d \tilde{s}_{2}^{\prime}, \\
& \mu_{\tilde{s}_{1}}=g_{0, \tilde{s}_{1}}^{*} / g_{i, \tilde{r}_{1}}^{*}, \mu_{\tilde{s}_{2}}=g_{0, \tilde{s}_{2}}^{*} / g_{i, \tilde{s}_{2}}^{*}, \\
& G_{\tilde{s}_{1}}=\frac{g_{0, \tilde{s}_{1}}^{*}+g_{i, \tilde{s}_{1}}^{*}}{\lambda_{c}}, G_{\tilde{s}_{2}}=\frac{g_{0, \tilde{s}_{2}}^{*}+g_{i, \tilde{s}_{2}}^{*}}{\lambda_{l}}, G=\sqrt{G_{\tilde{s}_{1}} G_{\tilde{s}_{2}}}
\end{aligned}
$$

Here $C_{m}$ is the capacitance of smooth muscle; $g_{i, \tilde{r}_{1}}^{*}, g_{i, \tilde{s}_{2}}^{*}, g_{0, \tilde{s}_{1}}^{*}, g_{0, \tilde{r}_{2}}^{*}$ are the maximal intracellular (i) and interstitial space (0) conductivities of the longitudinal and circular muscle layers; $M_{v s}$ is the membrane volume-to-surface ratio. The total ion current, $I_{\text {ionic }}^{*}$, is defined as

$$
I_{\text {ionic }}^{*}=\bar{g}_{\mathrm{Na}} m^{* 3} h^{*}\left(\varphi_{(c, l)}-\bar{\varphi}_{\mathrm{Na}}\right)+\bar{g}_{\mathrm{K}} n^{* 4}\left(\varphi_{(c, l)}-\bar{\varphi}_{\mathrm{K}}\right)+\bar{g}_{\theta}\left(\varphi_{(c, l)}-\bar{\varphi}_{\theta}\right),
$$

where $\bar{g}_{\mathrm{Na}}, \bar{g}_{\mathrm{K}}, \bar{g}_{\theta}$ are the maximal conductances and $\bar{\varphi}_{\mathrm{Na}}, \bar{\varphi}_{\mathrm{K}}, \bar{\varphi}_{\theta}$ are the reversal potentials of $\mathrm{Na}^{+}, \mathrm{K}^{+}$, and $\mathrm{Cl}^{-}$currents. The dynamics of change in the probability variables $m^{*}, h^{*}$, and $n^{*}$ of opening of the ion gates are obtained from the solution of the first-order equation

$$
\frac{d \eta}{d t}=a_{\eta}^{*}(1-\eta)-\beta_{\eta}^{*} \eta, \quad\left(\eta=m^{*}, h^{*}, n^{*}\right)
$$

The activation, $\alpha_{\eta}^{*}$, and deactivation, $\beta_{\eta}^{*}$, parameters satisfy the empirical relations

$$
\begin{aligned}
& a_{m}^{*}=0.005\left(\varphi_{(c, l)}-\bar{\varphi}_{m}\right) /\left[\exp 0.1\left(\varphi_{(c, l)}-\bar{\varphi}_{m}\right)-1\right], \\
& \beta_{m}^{*}=0.2 \exp \left(\varphi_{(c, l)}+\bar{\varphi}_{m}\right) / 38, \\
& a_{h}^{*}=0.014 \exp \left[-\left(\bar{\varphi}_{h}+\varphi_{(c, l)}\right) / 20\right], \\
& \beta_{h}^{*}=0.2 /\left[1+\exp 0.2\left(\bar{\varphi}_{h}-\varphi_{(c, l)}\right)\right], \\
& a_{n}^{*}=0.006\left(\varphi_{(c, l)}-\bar{\varphi}_{n}\right) /\left[\exp 0.1\left(\varphi_{(c, l)}-\bar{\varphi}_{n}\right)-1\right], \\
& \beta_{n}^{*}=0.75 \exp \left(\bar{\varphi}_{n}-\varphi_{(c, l)}\right) .
\end{aligned}
$$

The dynamics of the propagation of the electrical wave, $\varphi_{c}$, along the isotropic circular smooth muscle syncytium is given by

$$
C_{m} \frac{\partial \varphi_{c}}{\partial t}=\frac{M_{v s}}{1+\mu_{\tilde{s}_{1}}}\left\{\frac{\partial}{\partial \tilde{s}_{1}}\left(\frac{g_{0, \tilde{s}_{1}}^{*}}{\lambda_{c}} \frac{\partial \varphi_{c}}{\partial \tilde{s}_{1}}\right)+\frac{\partial}{\partial \tilde{s}_{2}}\left(\frac{g_{0, \tilde{s}_{1}}^{*}}{\lambda_{l}} \frac{\partial \varphi_{c}}{\partial \tilde{s}_{2}}\right)\right\}-I_{\text {ionic }}^{*},
$$


where the above-mentioned abbreviations and relationships for $I_{\text {ionic }}^{*}$, eqns (11-13), are used.

The gastric pressure, $p$, changes according to the adiabatic law

$$
p=p_{0} \Delta V^{1.41},
$$

where $p_{0}$ is the meaning of $p$ at $t=0 ; \Delta V=V_{0} / V$ is the ratio of a current intraluminal volume, $V$, to its initial value, $V_{0}$.

The cardiac and pyloric ends of the organ are clamped

$$
t>0: x\left(\tilde{s}_{1}, \tilde{s}_{2}\right)=y\left(\tilde{s}_{1}, \tilde{s}_{2}\right)=z\left(\tilde{s}_{1}, \tilde{s}_{2}\right)=0 .
$$

The initial conditions assume that the stomach is in the resting state

$$
t=0: \quad v_{r}=v_{s}=v_{z}=0 ; \varphi_{l}=\varphi_{c}=0 .
$$

Excitation to the system is provided by electrical discharges of the pacemaker cell that are modeled as an input impulse of a given amplitude, $\varphi_{0}$, and duration $t_{d}$

$$
\begin{gathered}
\varphi_{l}\left(\tilde{s}_{1}, \tilde{s}_{2}\right)= \begin{cases}\varphi_{0}, & 0<t \leq t_{d} \\
0, & t>t_{d},\end{cases} \\
\varphi_{c}\left(\tilde{s}_{1}, \tilde{s}_{2}\right)= \begin{cases}\varphi_{0}, & t_{i}<t \leq t_{d}+t_{i} \\
0, & t>t_{d}+t_{i},\end{cases}
\end{gathered}
$$

where $t_{i}\left(t_{i} \geq 0\right)$ is the time interval between discharges on the longitudinal and circular smooth muscle layers.

The combined nonlinear system of partial and ordinary differential equations was solved numerically. Hybrid finite difference and finite element methods of second-order accuracy, with respect to spatial and time variables, were employed. The numerical algorithm was implemented in the ABS Technologies $^{\circledR}$ computational platform. The accuracy of the algorithm and robustness of the platform have been evaluated extensively through multiple numerical experiments (different levels of discretization and time steps of integration, etc.) and the variation of parameters and constants of the model.

\section{RESULTS OF SIMULATIONS}

\subsection{Inflated stomach}

Consider a soft biological shell in the state of dynamic equilibrium. It is supported by intraluminal pressure $p=20 \mathrm{kPa}$. The strain distribution in the wall of the bioshell is shown in Fig. 1. The maximal elongation of the longitudinal muscle fibers, $\lambda_{l}=1.35$, is observed in the cardio-fundal region along the greater curvature of the organ. A part of the fundus bordering the body experiences the maximal biaxial extension with $\lambda_{l}=1.35, \lambda_{c}=1.04$ in that area. The cardia and the body of the stomach along the lesser curvature, both undergo biaxial deformation with $\lambda_{l}=1.01, \lambda_{c}=1.24$, while the antrum-pyloric region along the greater curvature is under uniaxial strain, $\lambda_{l}=0.5, \lambda_{c}=1.16$. Negative circumferential deformations are registered in the cardia and the pylorus. Thus, the fundus and the body of the organ undergo biaxial distension and the cardia and antrum-pyloric areas are subjects to uniaxial elongation.

Analysis of the total force distribution in the bioshell demonstrates that $\max T_{l}=9.3 \mathrm{mN} / \mathrm{cm}, T_{c}=$ $14.5 \mathrm{mN} / \mathrm{cm}$ are recorded in the fundus and the body. The maximum $T_{c}=34.7 \mathrm{mN} / \mathrm{cm}$ is registered in a small area of the body along the lesser curvature of the stomach. In the antrum and the lower cardia, total forces of average intensity $T_{l}=4.1 \mathrm{mN} / \mathrm{cm}, T_{c}=14.5 \mathrm{mN} / \mathrm{cm}$ are observed. Again, in the pylorus and the cardia the total force in the circumferential direction equals zero, while in the longitudinal direction, $T_{l}=3.0 \mathrm{mN} / \mathrm{cm}$. The proximal part of the cardia remains unstressed with $T_{l}=T_{c}=0$. 

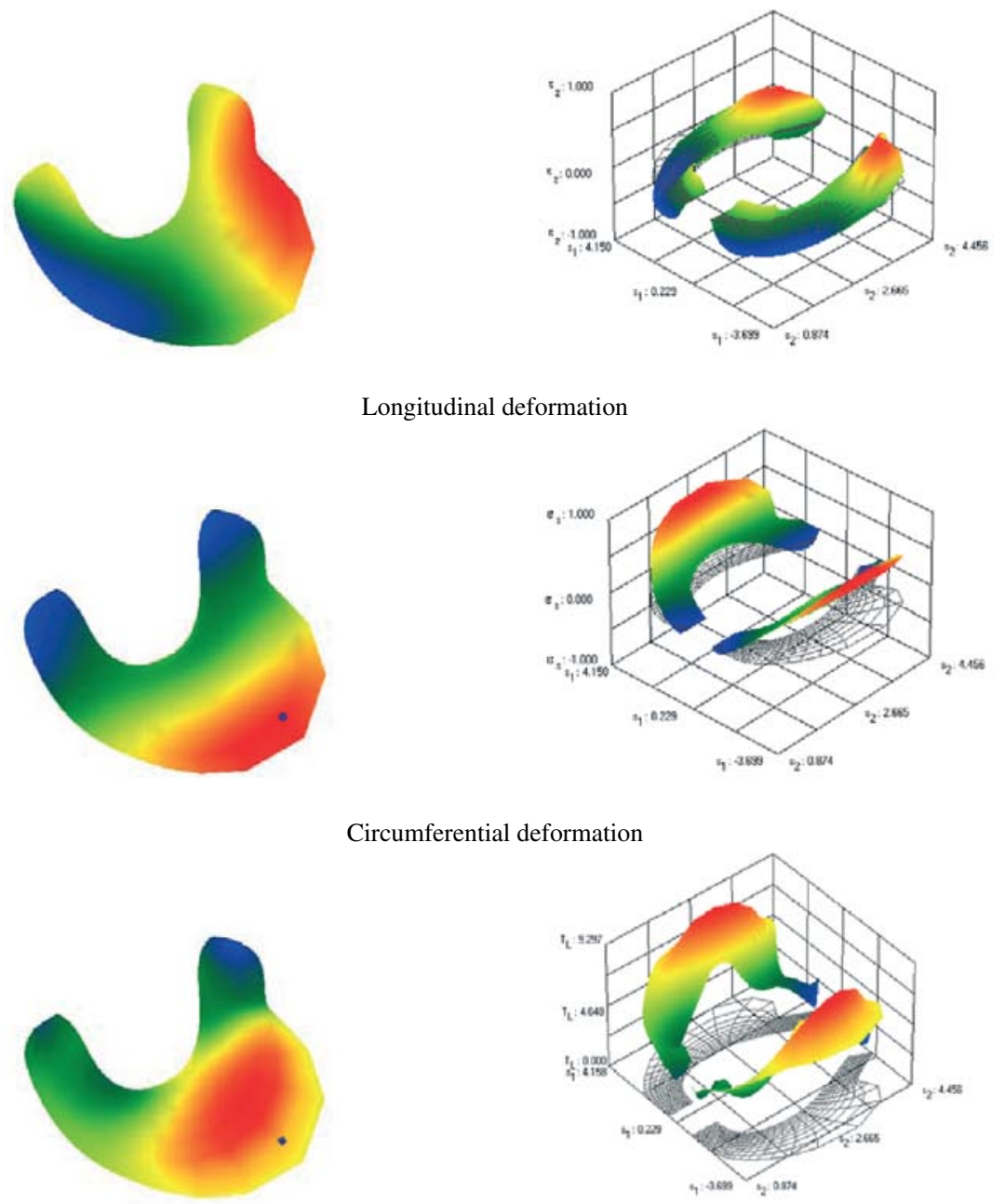

Total force in the longitudinal syncytium
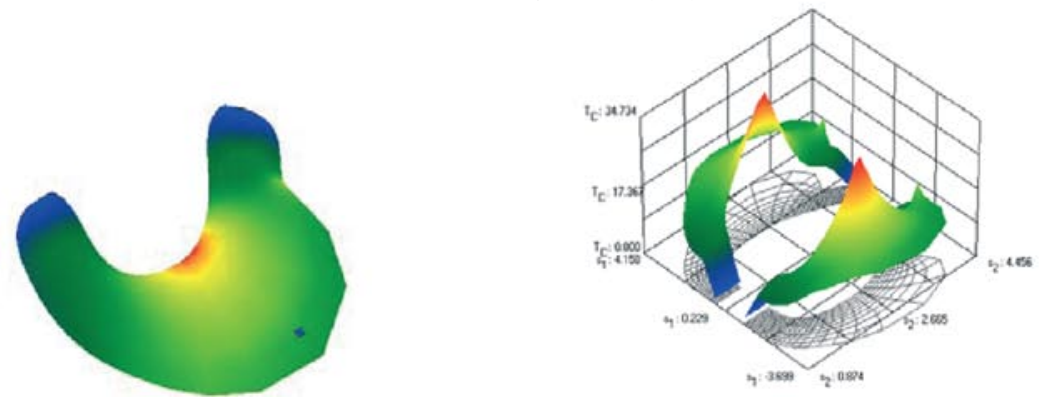

Total force in the circumferential syncytium

Figure 1: Static distribution of deformations and total forces in the longitudinal and circumferential smooth muscle syncytia of the inflated human stomach. Hereafter, results of simulations are presented on the entire stomach and the open surface envelope, respectively. 


\subsection{Electromechanical wave activity}

Consider a case of two identical pacemaker cells located on the longitudinal and circular smooth muscle layers in the upper body along the greater curvature of the stomach. Let the cells discharge simultaneously multiple impulses $(n=5)$ of amplitude $\varphi_{0}=100 \mathrm{mV}$ and duration $t_{d}=0.1 \mathrm{~s}$. The activation of ion channels on the membrane of muscle causes the generation of the waves of depolarization of amplitude $\varphi_{(l, c)}=65-70 \mathrm{mV}$ (Fig. 2). The velocity of the propagation of excitation varies between the syncytia and regions of the organ. Thus the wave $\varphi_{l}$ quickly spreads within the longitudinal muscle fibers to encase a narrow zone within the anterior surface of the organ $(t=0.8 \mathrm{~s})$. The wave $\varphi_{l}$ sustains short wave-length and a constant amplitude $18 \mathrm{mV}$ throughout $2.0<t<5.6 \mathrm{~s}$. The high level of depolarization is observed in the pyloric region where $\varphi_{l}=25.2 \mathrm{mV}$ is registered.
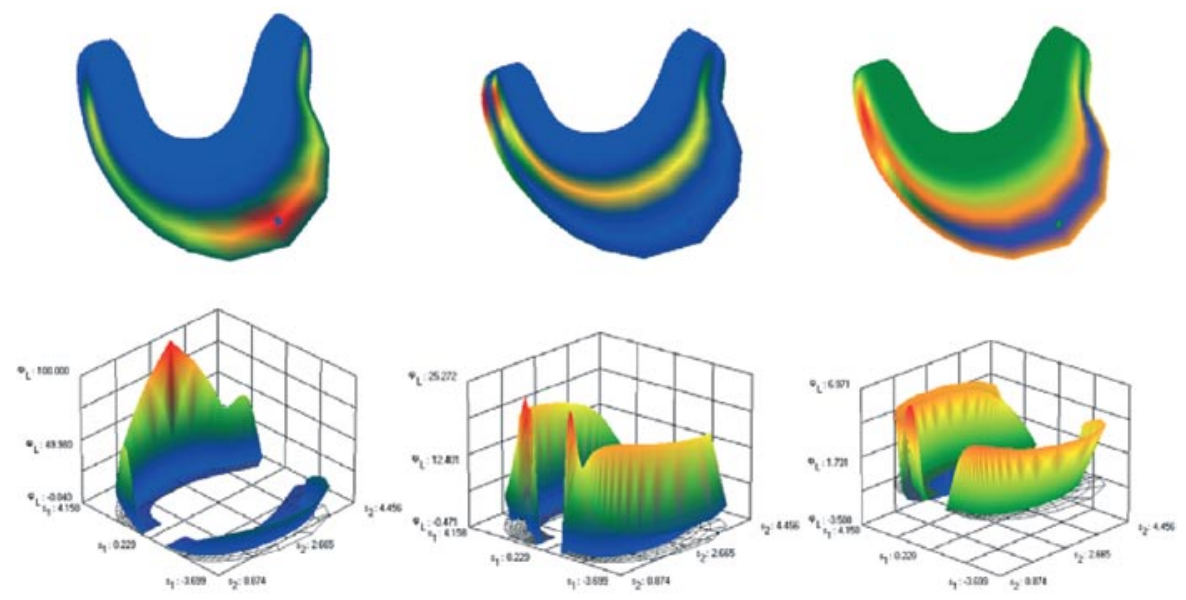

Longitudinal syncytium
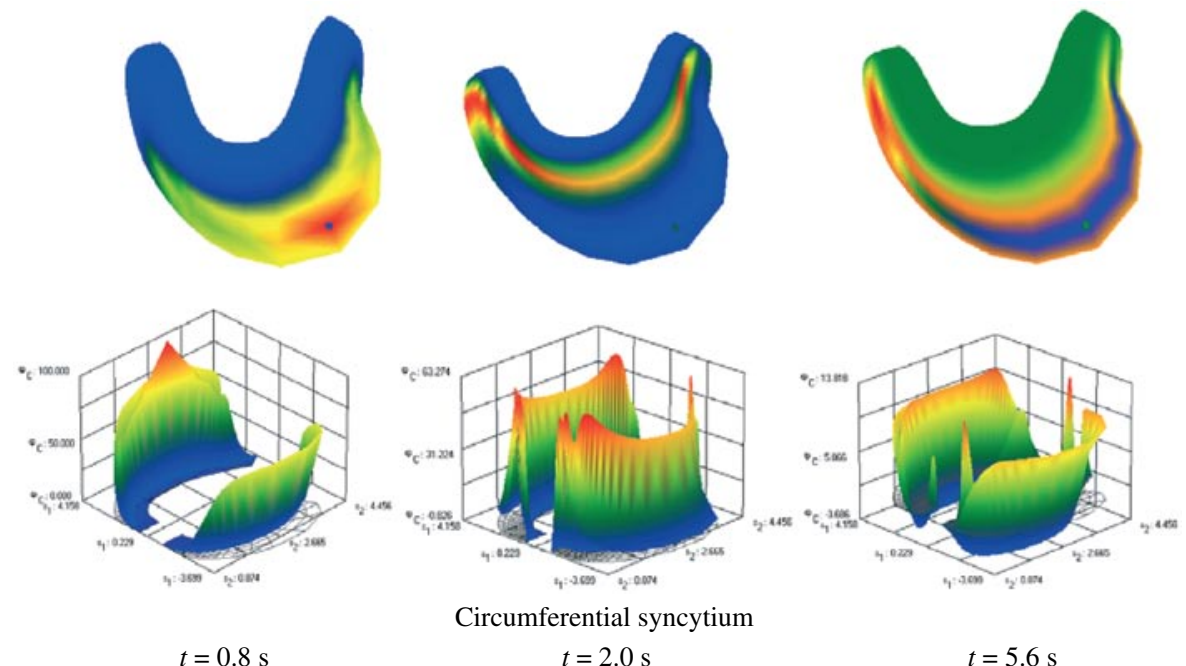

$$
t=0.8 \mathrm{~s}
$$

$$
t=2.0 \mathrm{~s}
$$

$$
t=5.6 \mathrm{~s}
$$

Figure 2: Dynamics of the propagation of the electrical wave of depolarization within different layers of the human stomach wall as a response to high frequency discharges of the pacemaker cell located in the body of the organ along its greater curvature. 
The propagation of the wave $\varphi_{c}$ within the isotropic circular smooth muscle layer is faster compared to $\varphi_{l}$. Thus, at $t=0.8 \mathrm{~s}$ nearly a half of the anterior and posterior surfaces of the bioshell undergo even depolarization, $60<\varphi_{c}<66.5(\mathrm{mV})$. The excitation quickly extends towards the lesser curvature. The cardiac and pyloric regions experience extensive depolarization with a $\max \varphi_{c}=63.3$ $\mathrm{mV}$. At $t=5.6 \mathrm{~s}$ the longitudinal and circular smooth muscle syncytia of the stomach show a similar pattern in the distribution of depolarization with the average amplitude $\varphi_{l} \approx 3.7 \mathrm{mV}, \varphi_{c} \approx 7.5 \mathrm{mV}$.

The excitation of voltage-dependent calcium channels on the membrane of smooth muscle syncytia results in a rapid influx of $\mathrm{Ca}^{2+}$ ions and rise in the free cytosolic $\mathrm{Ca}^{2+}$ concentration to $0.48 \mu \mathrm{M}$. The above lead to the activation of contractile proteins and the generation of active forces of contraction. There is a smooth distribution of active forces of contraction in the fundus and the body of the stomach where $T_{l}^{a}=6.1 \mathrm{mN} / \mathrm{cm}$ and $T_{c}^{a}=7.4 \mathrm{mN} / \mathrm{cm}$ are generated during $0<t \leq 5.0 \mathrm{~s}$ (Fig. 3). The cardia and the antrum-pyloric regions generate less intense forces, $T_{l}^{a}=3.0-3.6 \mathrm{mN} / \mathrm{cm}$ and $T_{c}^{a}=3.7-4.4 \mathrm{mN} / \mathrm{cm}$. At $t=5.6 \mathrm{~s}$ of the dynamic process the redistribution in stresses occurs with the high level of contraction, $\max T_{l}^{a}=7.9 \mathrm{mN} / \mathrm{cm}$, seen in the body of the organ. There is a concentration in active force development in the circular smooth muscle layer in the body along its lesser curvature with $\max T_{c}^{a}=10.5 \mathrm{mN} / \mathrm{cm}$. The rest of the fundus and the body experience even contractions, $T_{l}^{a}=6.9, T_{c}^{a}=8.4(\mathrm{mN} / \mathrm{cm})$.

At $t=5.6 \mathrm{~s}$ the earlier existing zone of uniaxial stretching and wrinkling in the antrum-pyloric regions along the greater curvature has expanded to the lower part of the body. Sustained unidirectional longitudinal elongations persist in the cardia. However, a new zone of uniaxial stretch oriented along the longitudinal axis is generated along the lesser curvature of the bioshell.

The pattern of total force distribution is similar to that observed at the state of dynamic equilibrium. There is an increase in the intensity of forces, which is consistent with the generation of active forces of contraction by the syncytia (Fig. 3). Thus, $\max T_{l}=15.1 \mathrm{mN} / \mathrm{cm}, T_{c}=22.4 \mathrm{mN} / \mathrm{cm}$ are seen in the body, and $T_{l}=2.7 \mathrm{mN} / \mathrm{cm}, T_{c}=5.3 \mathrm{mN} / \mathrm{cm}$ in the cardia and the pylorus of the stomach.

\subsection{Time lag in firing of pacemakers}

Consider the effect of time delay $\left(t_{i}\right)$ in discharges of pacemaker cells on the dynamics of the force elongation development in the bioshell. The number of impulses, their amplitude and duration correspond to the experimental conditions as described above. The pacemaker located in the longitudinal muscle syncytium fires first, followed by a discharge of the pacemaker on the circular muscle syncytium at $t_{i}=0.75 \mathrm{~s}$.

Results of simulations show that there are no significant differences in the intensity of force development. However, the delay in activation of the circular smooth muscle layer results in early wrinkling $(t=0.8 \mathrm{~s})$ in the body along the lesser curvature which persists throughout. The delayed generation of contractions in the circular smooth layer results in a reciprocal contraction-relaxation relation between the two bisyncytia. The pattern of electromechanical activity resembles peristalsis, i.e. a propagating wave of contraction-relaxation that satisfies the condition of reciprocity (Fig. 4).

\subsection{Multiple pacemakers}

Consider three identical pacemaker cells spatially distributed along the anterior wall of the bioshell. The leading pacemaker is located in the upper part of the body, and the other two are positioned in the antrum and the pyloric regions of the stomach, respectively. The cells discharge impulses of amplitude $\varphi_{0}=100 \mathrm{mV}$ and duration $t_{d}=0.1 \mathrm{~s}$. The time lag between the beginning of discharges of pacemakers is constant, $t_{i}=0.75 \mathrm{~s}$.

Results of simulations demonstrate that at $t=1.2 \mathrm{~s}$ the entire organ undergoes higher levels of uniform depolarization, $\varphi_{l} \approx \varphi_{c} \approx 55 \mathrm{mV}$ (Fig. 5). This pattern of excitation persists till $t=7 \mathrm{~s}$. 
100 N.R. Akhmadeev \& R. Miftahof, Int. J. of Design \& Nature and Ecodynamics. Vol. 5, No. 2 (2010)
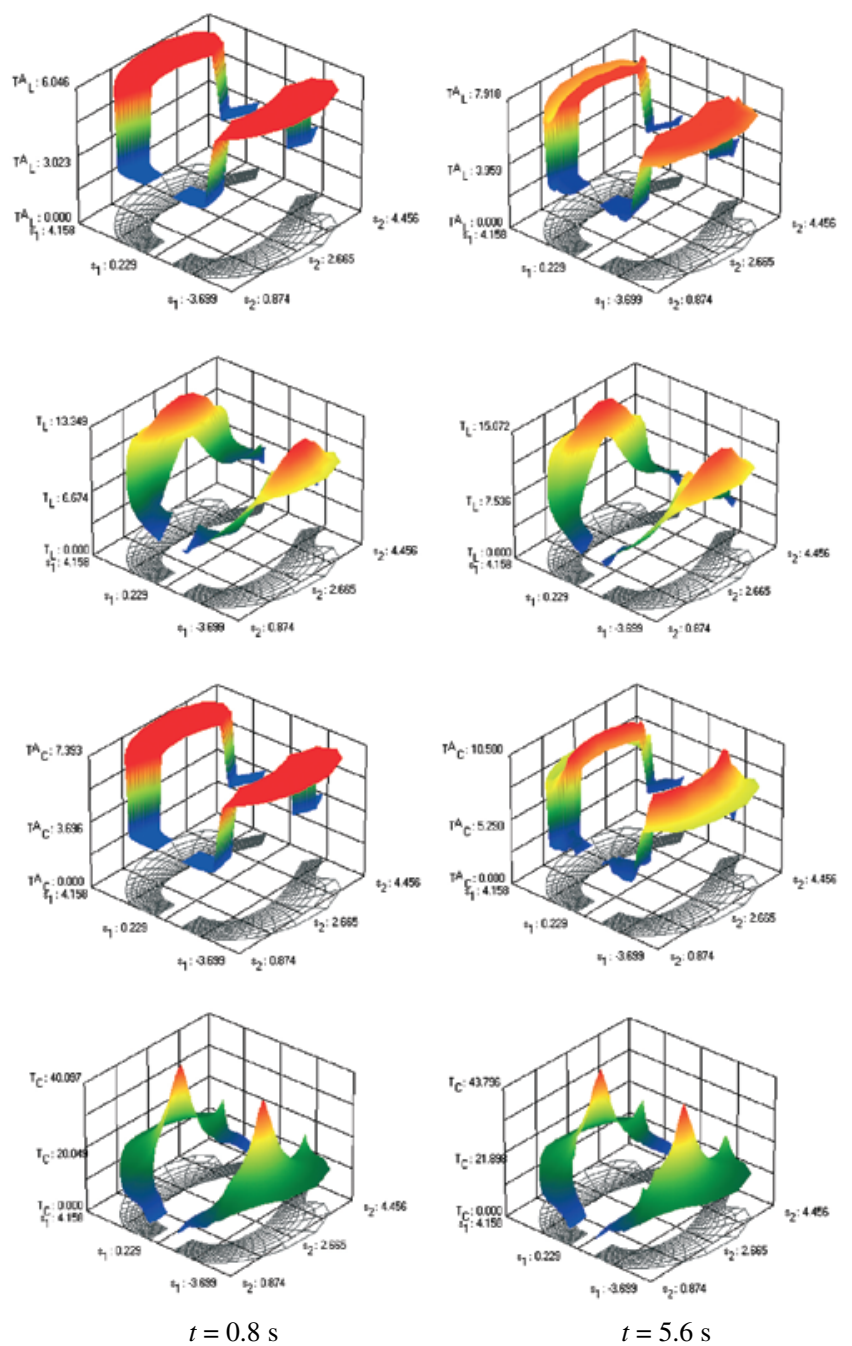

Figure 3: Development of active and total forces in the stomach as a result of the electromechanical coupling process in the myogenic syncytia.

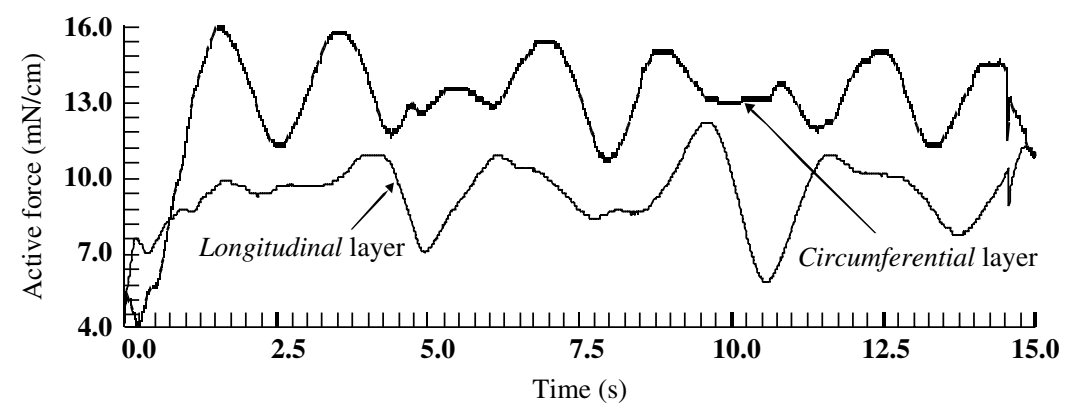

Figure 4: Changes in the active force dynamics in the longitudinal and circumferential smooth muscle layers at a "'control' point in the body of the anterior surface of the human stomach. 
N.R. Akhmadeev \& R. Miftahof, Int. J. of Design \& Nature and Ecodynamics. Vol. 5, No. 2 (2010) 101
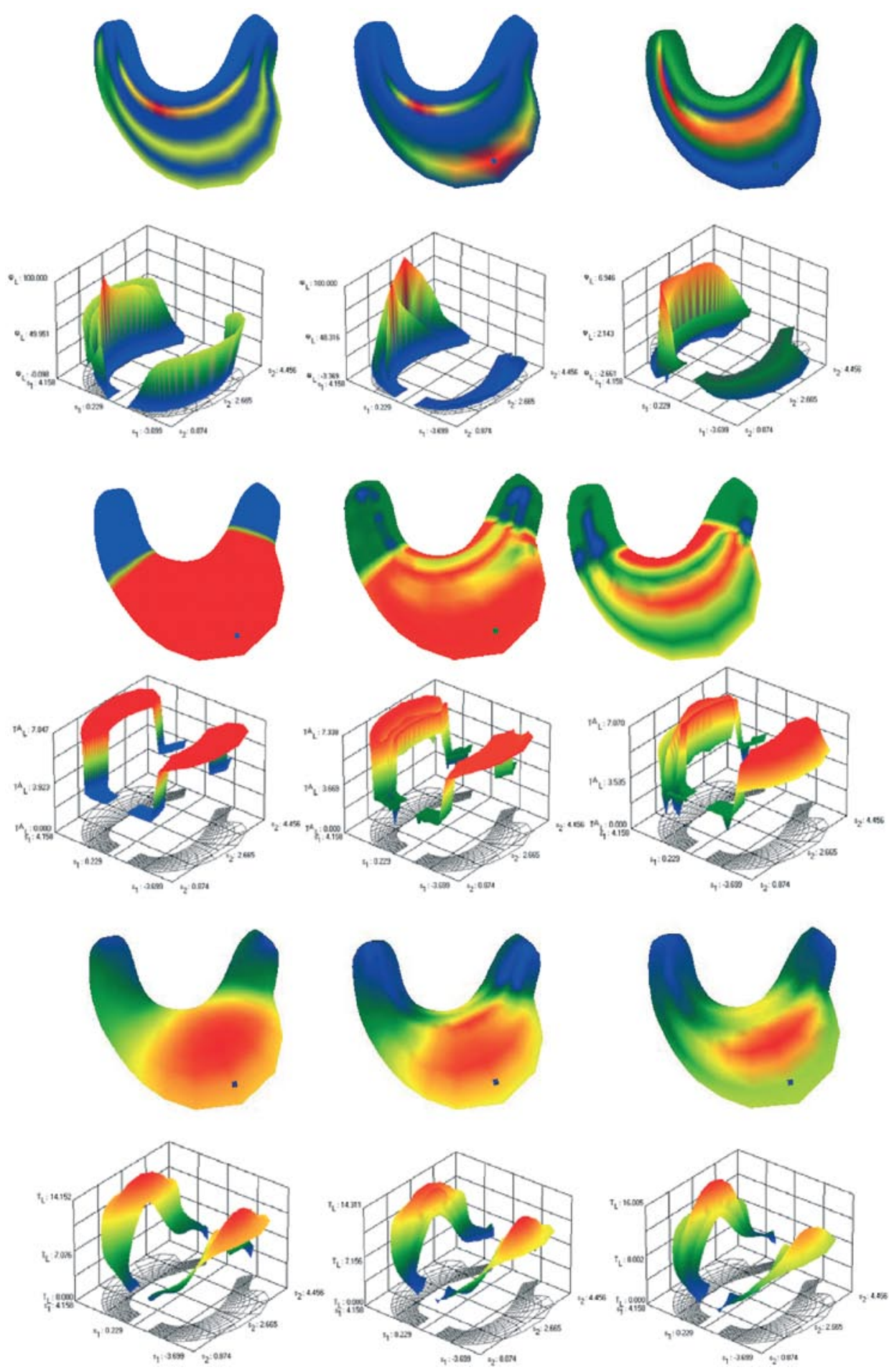

Figure 5: Dynamics of electromechanical responses of the soft biological shell - the human stomach, to high frequency stimulation by multiple pacemakers located in different regions of the organ. 
102 N.R. Akhmadeev \& R. Miftahof, Int. J. of Design \& Nature and Ecodynamics. Vol. 5, No. 2 (2010)
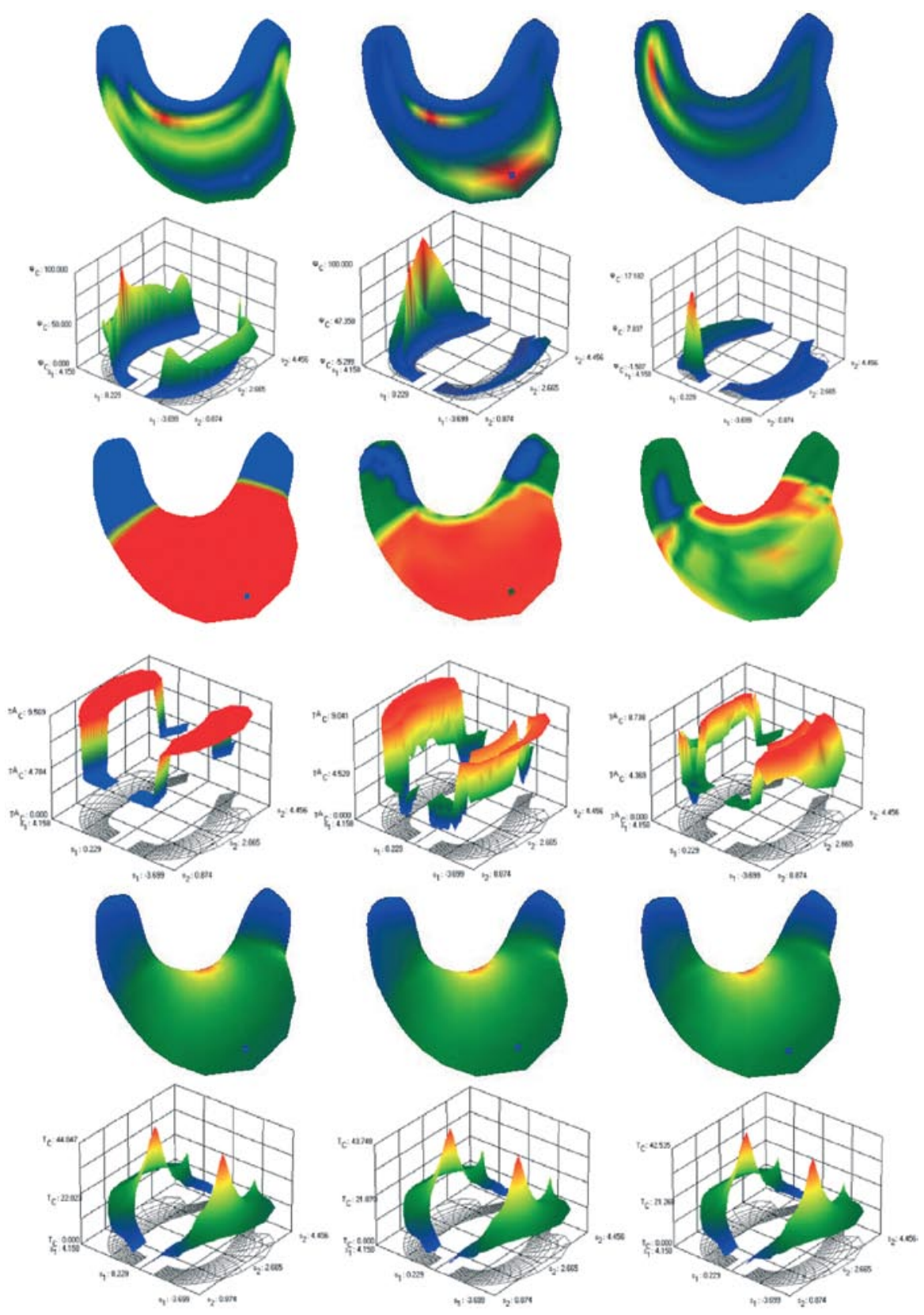

$t=1.2 \mathrm{~s}$

$t=7.0 \mathrm{~s}$

$t=10.8 \mathrm{~s}$

Figure 5: (continued)

For $t=10.8 \mathrm{~s}$ the longitudinal smooth muscle layer of the anterior wall of the stomach in the projection of the body, antrum and pylorus remains excited, $\varphi_{l} \approx 6.8 \mathrm{mV}$, while the circumferential layer experiences hyperpolarization $\varphi_{c} \approx-1.2 \mathrm{mV}$. There is an area of high depolarization in the pyloric region where $\varphi_{c} \approx 17.1 \mathrm{mV}$ is recorded. 
In the time interval $1.2 \leq t \leq 7$ (s) the longitudinal and circumferential smooth muscle layers of the fundus, body and antrum generate smooth forces of contraction, $T^{u}{ }_{l}=7.4 \mathrm{mN} / \mathrm{cm}, T^{u}{ }_{c}=9.2 \mathrm{mN} / \mathrm{cm}$. Suffice it to note, that at the beginning of the process, $t=1.2 \mathrm{~s}$, the entire organ is stress-strained biaxially. At $t=7 \mathrm{~s}$ the creases oriented longitudinally occur in the cardia and pyloric regions along the lesser curvature. With the redistribution of active forces, $T^{a}{ }_{c}$, within the circular syncytium, $t>$ $10 \mathrm{~s}$, they disappear. The most intense contractions are registered in the body at the lesser curvature, $\max T_{l}^{u}=7, \max T_{c}^{u}=8.7(\mathrm{mN} / \mathrm{cm})$.

The involvement of the connective tissue network in the dynamics of load distribution provides 'smooth' deformation in all regions of the stomach and prevents the occurrence of gradients in $T_{(c, l)}\left(\tilde{s}_{1}, \tilde{s}_{2}, t\right)$. Thus, the bioshell passes through various stress-strain states without developing overstressed zones (Fig. 5). There is a persistent small area in the body along the lesser curvature where a $\max T_{c}=44 \mathrm{mN} / \mathrm{cm}$ is registered. Other anatomical regions experience even biaxial stress states of average intensities: $T_{l}=T_{c}=2-3 \mathrm{mN} / \mathrm{cm}$ in the cardia, $T_{l}=6.5, T_{c}$ $=9.4(\mathrm{mN} / \mathrm{cm})$ in the fundus, $T_{l}=10, T_{c}=15(\mathrm{mN} / \mathrm{cm})$ in the body, and $T_{l}=5, T_{c}=9(\mathrm{mN} / \mathrm{cm})$ in the antrum-pylorus.

\section{DISCUSSION}

The main hurdle that every computational modeler faces during the development of a biomechanical model of a living object is the paucity of reliable information about mechanical characteristics of biological tissues. Until now, there have been only a few correctly planned and executed experimental studies that are dedicated to the analysis of the human stomach from the quantitative mechanical perspective. The first uniaxial and biaxial tensile tests on tissue samples collected from various regions of the organ including the cardia, the fundus, the body, the antrum and the pylorus, were reported by Miftakhov [26-30]. The specimens were excised along two mutually orthogonal directions - longitudinal and circumferential, to study the axes of anisotropy. Results of investigations revealed that: (1) the wall of the stomach, like other soft tissues, exhibits nonlinear transversely anisotropic mechanical properties; (2) it undergoes large deformations $\approx 160-180 \%$; (3) there are regional and wall differences in mechanical properties, e.g. the anterior wall is more elastic and stretchable compared to the posterior wall; (4) there is stiffening of the biocomposite related to age changes. The experimentally obtained data on force-ratio of elongation served as a basis for the construction of phenomenological constitutive relations (3), (4), (8). They successfully described and offered robust estimates of the mechanical behavior of the stomach. Recent studies by different research groups performed on cadaveric, surgically removed human stomachs and healthy volunteers confirmed nonlinear anisotropic mechanical properties of the tissue and their regional dependence [31].

Miftakhov [32] was the first to formulate a biomechanical model of the organ as a soft multilayer biocomposite shell. Under general assumptions of curvilinear orthotropy, physical and geometrical nonlinearity, numerical and experimental investigations of the dynamics of stress-strain distribution in the organ under simple and complex loadings were performed. The dynamics of the development of uniaxial stress-strained states in the cardia and pylorus as a function of intraluminal pressure was demonstrated computationally and supported experimentally. Results offered a valuable insight into the mechanism of blunt abdominal trauma with rupture of the anterior wall of the stomach and gave a biomechanical explanation for the Mallory-Weiss syndrome. It was thought previously that atrophic changes in the gastric mucosa and submucous layer were responsible for longitudinal tears in the cardia-fundal region and life threatening intragastric bleeding. The model study proved that the anatomical structure and configuration of the stomach per se makes these regions more susceptible, than the others, to linear submucous breaks and ruptures. 
The presented model is an extension of the previous model. It incorporates myoelectrical properties of the longitudinal and circular smooth muscle syncytia along with mechanical nonlinearity of the wall of the stomach. Special emphasis in the study was given to the questions of electromechanical coupling in smooth muscle syncytia and cable electromechanical wave activity.

Gastric motility is a result of electromechanical coupling that occurs at the cellular level and is manifested in the form of electromechanical wave activity within the organ wall. In the current study we restricted our attention to the cable, rather than to more general oscillatory, properties of smooth muscle syncytia. The network of interstitial cells of Cajal - pacemakers of gastric motility, was substituted by a single cell or a set of spatially distributed cells. They discharged a priori known electrical impulses. Even with these constructive simplifications, the model reproduced and predicted: (1) patterns of the propagation of the wave of excitation within the electrically anisotropic longitudinal and electrically isotropic circular smooth muscle syncytia; (2) small isolated contraction waves in the two smooth muscle syncytia and nonpropagating tonic simultaneous contractions of both muscle layers superimposed on small contractions; (3) the development of wrinkles in the cardia, the body along the lesser curvature and the pylorus, and (4) high levels of tension in the body of the stomach along the lesser curvature.

A note of caution though should be made at this point of discussion. Care should be taken in transferring the results of simulations to explain real biomechanics of the human stomach. One has to bear in mind that the biological plausibility of the model is constrained by the model assumptions, despite the fact that the theoretical results resemble qualitatively patterns of electrical and mechanical activity that are observed in mainly animal studies in vivo and in vitro. At the moment there is no direct affirmative experimental evidence obtained on human subjects to run a detailed quantitative evaluation and comparison of the computational data.

Unfortunately, it is still a common practice in the community of modelers to employ the system of Navier-Stokes equations when modeling the stomach as a 'shell' structure. Using commercially available software and highly flexible graphical tools they manage to fit results of numerical simulations to experimental data. The approach is utterly incorrect and results bring confusion, rather than provide solutions, to urgent clinical problems related to gastric function. Thus, it is erroneous to claim the dominant role of antral contraction wave activity on gastric fluid motions, based on the results of computer simulations of a flow caused by prescribed indentations of the surface boundaries $[9,10]$. The latter is supposed to represent a two dimensional model of the stomach. And it is not surprising that the numerical method, that predetermines the desired patterns of flow, produced the results that resembled those observed in magnetic resonance imaging studies. An adequate mathematical model of the above phenomenon should have comprised of the combined system of the equations of motion of the bioshell - the stomach, and Navier-Stokes equations to model gastric content - thick semi-fluid mass of partly digested food and secretions (chyme). Suffice it to note, that until now no attempts have been made to describe the chyme as a chemically reactive medium. Also, in view of the fundamental mechanical property of the tissue, its softness, it is unwise to argue the dependence of tension-strain properties on the radii of curvature of the stomach [33]. It is the responsibility of an applied mathematician, a computer scientist, and a mechanical engineer to suggest an adequate descriptor and to give a rigorous mathematical formulation of the model.

Although the proposed model of the human stomach as a soft biological shell has a very limited biomedical value, at the moment, it is mathematically sound and is based on the accurate extension of general laws and hypotheses of the mechanics of thin soft shells. It incorporates electrophysiological and morphological data of the structure and function of the organ and reproduces quantitatively and qualitatively the dynamics of electromechanical wave activity and stress-strain distribution in it. It can serve as a starting point for further expansions and biological improvements. 


\section{FUTURE DIRECTIONS}

As an example of further model improvements and development consider the following biologically plausible modifications.

\subsection{Oscillatory myoelectrical activity}

Incorporation of oscillatory myoelectrical phenomenon will complete the model of electromechanical conjugation with the generation and propagation of electromechanical waves in the stomach. The model description should be based on: (1) the Hodgkin-Huxley formalism in mathematical modeling of the dynamics of L- and T-type $\mathrm{Ca}^{2+}$ channels, $\mathrm{Ca}^{2+}$-activated $\mathrm{K}^{+}$, selective $\mathrm{K}^{+}, \mathrm{Na}^{+}$, leak $\mathrm{Cl}^{-}$, and nonselective cation channels, and (2) the concept of spatially distributed weakly connected oscillators. We should point up the use of Hodgkin-Huxley equations in the model that will become essential in future investigations of the effects of pharmacological compounds on gastric motility.

\subsection{Neuronal networks and pacemaker activity}

The myenteric and submucous nervous plexi are contained within the wall of the stomach and constitute only the first level in the hierarchy of neuronal systems that regulate gastric functions. The plexi are formed of multiple ganglia of the primary sensory, inter- and motor neurons, arranged in a planar neuronal network. Recent research has suggested another intrinsic network of interstitial cells of Cajal provides a modulatory signaling to the plexi. We possess ample experimental data on histomorphology, electrophysiology and neuropharmacology of neurons and cell of Cajal to construct a detailed mathematical model of the intrinsic regulatory neuronal network.

\subsection{Electrochemical coupling and neurotransmitters}

Neurons form chemical synapses within plexi. Immunohistochemical and radiolabeling studies revealed that neurons store and can release more than one neurotransmitter substance at a nerve terminal. The neurotransmitters may interact at many levels of their synthesis, storage, release, degradation and postsynaptic actions. Particular combinations of such substances correlate with specific functional classes of nerves. Mathematical modeling of the electrochemical coupling phenomenon - the process of transformation of chemical signals into electrical signals, is necessary for the full investigation of the effects of neurotransmitter co-localization for synaptic transmission. It will have enormous impact on the ability of the model to simulate and to predict the effects of different classes of drugs on gastric function.

\subsection{Brain-stomach axis}

The intramural nervous plexi function independently and in concert with the central nervous system. They communicate with the central nervous system (brain) through the sympathetic and parasympathetic nervous divisions of the autonomic nervous system. In the sympathetic nervous system, there are ganglia situated close to the stomach which participate in the control of contractions. These ganglia are connected to the spinal cord and to higher centers in the brain. The parasympathetic innervation is provided by the vagus nerve. It is of utmost importance to incorporate the above multilevel regulatory interactions in the model of the stomach. 


\subsection{Feedback regulation}

The fact that gastric motility in vivo changes in response to mechanical and chemical stimulation of the organ indicates the existence of feedback regulation in the system. These effects, commonly attributed to reflex pathways and hormonal (gastrin, cholecystokinin, secretin, etc.) actions, are considered to be critical to the normal operation of the stomach. The integration of such feedback control mechanisms into a model of the neuromuscular apparatus of the stomach constitutes a major problem in this area.

When the model is complete it will offer unique insights and will provide otherwise inaccessible information on intrinsic physiological processes of gastric function. Such an approach will have enormous implication for our understanding of mechanisms of certain diseases, advancing of diagnostic accuracy, and planning of therapeutic interventions.

\section{REFERENCES}

[1] Cowin, S.C., How is a tissue built? Transactions of ASME, Journal of Biomechanical Engineering, 122, pp. 553-559, 2000. doi:10.1115/1.1324665

[2] Humphrey, J.D., Continuum biomechanics of soft biological tissues. Proceedings of the Royal Society, Ser. A, 459, pp. 3-46, 2003.

[3] Hiroshi, Y., Strength of Biological Materials, Williams \& Wilkins, 1970.

[4] Miftakhov, R.N., Influence in the ion concentration changes on excitation propagation and mechanical response in smooth muscle. Mechanics of Biological Solids, The Academy of Sciences of the USSR, pp. 81-101, 1986 (in Russian).

[5] Miftakhov, R.N., Applications of the theory of soft thin shells in problems of biomechanics. Biomechanics: Problems and Investigations, Zinatne: Riga, Vol. VI, pp. 51-56, 1988.

[6] Pullan, A., Cheng, L., Yassi, R. \& Buist, M., Modelling gastrointestinal bioelectric activity. Progress in Biophysics and Molecular Biology, 85, pp. 523-550, 2004. doi:10.1016/j. pbiomolbio.2004.02.003

[7] Cheng, L., Komuro, R., Austin, T.M., Buist, M.L. \& Pullan A.J., Anatomically realistic multiscale models of normal and abnormal gastrointestinal electrical activity. World Journal of Gastroenterology, 13, pp. 1378-1383, 2007.

[8] Corrias, A. \& Buist, M.L., A quantitative model of gastric smooth muscle cellular activation. Annals of Biomedical Engineering, 35, pp. 1595-1607, 2007. doi:10.1007/s10439-007-9324-8

[9] Pal, A., Brasseur, J. \& Abrahamsson, B., A stomach road or "Magenstrasse" for gastric emptying. Journal of Biomechanics, 40, pp. 1202-1210, 2007. doi:10.1016/j.jbiomech.2006.06.006

[10] Pal, A., Indireshkumar, K., Schwizer, W., Abrahamsson, B., Fried, M. \& Brasseur, J.G., Gastric flow and mixing studied using computer simulation. Proceedings of the Royal Society, Ser. B, 271, pp. 2587-2594, 2004.

[11] Miftahof, R., Biomechanics of the Small Intestine, POSTECH University Press: Rep. Korea, 2005.

[12] Miftahof, R., Nam, H.G. \& Wingate D.L., Mathematical Modeling and Simulation in Enteric Neurobiology, World Scientific Publ.: Singapore, 2009

[13] Suzuki, H., Cellular mechanisms of myogenic activity in gastric smooth muscle. Japanese Journal of Physiology, 50, pp. 289-301, 2000. doi:10.2170/jjphysiol.50.289

[14] Hirst, D.G.S. \& Suzuki, H., Involvement of interstitial cells of Cajal in the control of smooth muscle excitability. Journal of Physiology, 576, pp. 651-652, 2006. doi:10.1113/jphysiol.2006.121178

[15] Koh, S.D., Ward, S.M., Tamas, O., Sanders, K.M. \& Horowitz, B., Conductances responsible for slow wave generation and propagation in interstitial cells of Cajal. Current Opinion in Pharmacology, 3, pp. 579-582, 2003. doi:10.1016/j.coph.2003.09.002 
[16] Carniero, A.A., Baffa, O. \& Oliveira, R.B., Study of stomach motility using relaxation of magnetic tracers. Physical and Medical Biology, 44, pp. 1691-1697, 1999. doi:10.1088/0031-9155/44/7/309

[17] Muraki, K., Imaizumi, Y. \& Watanabe, M., Sodium currents in smooth musclecells freshly isolated from stomach fundus of the rat and ureter of the guinea-pig. Journal of Physiology, 442, 351-375, 1991.

[18] Ou, Y., Strege, P., Miller, S.M., Makielski, J., Ackerman, M., Gibbons, S.J. \& Farrugia, G., Syntrophin gamma 2 regulates SCN5A gating by a PDZ domain-mediated interaction. Journal of Biological Chemistry, 278, pp. 1915-1923, 2003. doi:10.1074/jbc.M209938200

[19] Lyford, G.L., Strege, P.R., Shepard, A., Ou, Y., Ermilov, L., Miller, S.M., Gibbons, S.J., Rae, J.L., Szurszewski, J.H. \& Farrugia, G., Alpha 1C (Cav1.2) L-type calcium channel mediates mechanosensitive calcium regulation. American Journal of Physiology, Cell Physiology, 283, pp. C1001-C1008, 2002. doi:10.1016/j.coph.2003.06.010

[20] Lyford, G.L. \& Farrugia, G., Ion channels in gastrointestinal smooth muscle and interstitial cells of Cajal. Current Opinion in Pharmacology, 3, pp. 583-587, 2003.

[21] Dickens, E.J., Edwards, F.R. \& Hirst, G.D.S., Selective knockout of intramuscular interstitial cells reveals their role in the generation of slow waves in mouse stomach. Journal of Physiology, 531, pp. 827-833, 2001. doi:10.1111/j.1469-7793.2001.0827h.x

[22] Hennig, G.W., Hirst, G.D.S., Park, K.J., Smith, C.B., Sanders, K.M.,Ward, S.M. \& Smith, T.K., Propagation of pacemaker activity in the guinea-pig antrum. Journal of Physiology, 556, pp. 585-599, 2004. doi:10.1113/jphysiol.2003.059055

[23] Hirst, G.D.S., Garcia-London, A.P. \& Edwards, F.R., Propagation of slow waves in the guinea-pig gastric antrum Journal of Physiology, 571, pp. 165-177, 2006. doi:10.1113/jphysiol.2005.100735

[24] Bárány, M., Biochemistry of Smooth Muscle Contraction, Academic Press, 1996.

[25] Alvarez, W.C. \& Zimmermann, A., Movements of the stomach. American Journal of Physiology, 84, pp. 261-270, 1928.

[26] Miftakhov, R.N., Age changes of the 'quasi-equilibrium' module of elasticity of the human stomach. Shell Interactions with Fluids, The Academy of Sciences of the USSR, pp. 197-204, 1981 (in Russian).

[27] Miftakhov, R.N., Investigation of the human stomach tissue in uniaxial loading. Hydroelasticity of Shells, The Academy of Sciences of the USSR, pp. 163-171, 1983 (in Russian).

[28] Miftakhov, R.N., Experimental investigation of the stomach tissue in biaxial loading. Invest. in the Theory of Plates and Shells, Kazan State Univ. Press: Kazan, XVIII, pt. I, pp. 35-46, 1985 (in Russian).

[29] Miftakhov, R.N., Micromechanics of tissue fracture in uniaxial elongation. Shell Interactions with Fluids, The Academy of Sciences of the USSR, pp. 205-214, 1981 (in Russian).

[30] Miftakhov, R.N., Experimental investigations of the stomach under complex loading. Hydroelasticity of Shells, The Academy of Sciences of the USSR, pp. 172-181, 1983 (in Russian).

[31] Egorov, V.I., Schastlivtsev, I.V., Prut, E.V., Baranov, A.O. \& Turusov, R.A., Mechanical properties of the human gastrointestinal tract, Journal of Biomechanics, 35, pp. 1417-1425, 2002. doi:10.1016/S0021-9290(02)00084-2

[32] Miftakhov, R.N., Experimental and Numerical Investigations of Soft Shells, PhD Thesis, Kazan State University, Kazan, USSR, 1983 (in Russian).

[33] Liao, D., Gregersen, H., Hausken, T., Gilja, O.H., Mundt, M. \& Kassab, G., Analysis of surface geometry of the human stomach using real-time 3-D ultrasonography in vivo. Neurogastroenterology \& Motility, 16, pp. 315-324, 2004. doi:10.1111/j.1365-2982.2004.00522.x 